\title{
A Study on Fluctuations in Electromechanical Oscillation Frequencies of Power Systems
}

\author{
Bin Wang, Kai Sun \\ University of Tennessee \\ Knoxville, TN USA \\ bwang13@utk.edu \\ kaisun@utk.edu
}

\author{
Alberto Del Rosso, Evangelos Farantatos, Navin Bhatt \\ Electric Power Research Institute \\ Palo Alto, CA USA \\ adelrosso@epri.com \\ efarantatos@epri.com \\ nbhatt@epri.com
}

\begin{abstract}
This paper investigates the fluctuation phenomenon in the electromechanical oscillation frequency of a power system. Analysis on the power system swing equation explains that the oscillation frequency fluctuates around a central frequency due to the nonlinear system nature of a power system. A Phase-Plane Trajectory based method and the Prony method are used to track the real-time frequency fluctuation, based on which a realtime angular stability margin index is proposed for early warning of angular stability issues. That index is tested by case studies on a two-generator system and a 179-bus power system.
\end{abstract}

Index Terms-oscillation frequency; phase-plane trajectory; prony analysis; PMU; stability margin; synchrophasor

\section{INTRODUCTION}

Poorly damped oscillations in an interconnected power system may reduce the power transfer capabilities, result in detrimental economic and operational consequences and even threaten the angular stability of the system [1][2]. The computation of oscillation frequency (OF) is very important, especially for lightly damped modes. From the study on the synchronization phenomenon [3], the solution of the secondorder nonlinear differential equation, named van der Pol equation, has been shown to have a fluctuation in the $\mathrm{OF}$. When it comes to the power system, it is also possible for the generators' swing equations, which are essentially a combination of many second-order nonlinear differential equations, to have a fluctuation in the OF.

Nowadays, a majority of applications for computing power system frequency modes like Prony analysis [4], Subspace Identification method [5], Frequency Domain Decomposition [6] and Wavelet Transform based method [7] can provide accurate estimates of the $\mathrm{OF}$ and damping ratio of an oscillation mode. A recently proposed method based on Recursive Adaptive Stochastic Subspace Identification can give mode shapes besides the $\mathrm{OF}$ and damping ratio [8]. However, most of those methods need data over a certain time window to perform the computation and their results are actually average OF estimates over the time window and lack instantaneity. Therefore, when using some of those methods, e.g. Prony analysis, to track the OF in power systems, some dynamical phenomena of the OF due to the nonlinear nature of a power system might be missed.

This paper intends to reveal a fact that the OF fluctuates in a specific way following a disturbance due to the nonlinear nature of a power system. Such a fluctuation, if captured in real time by an effective OF estimation method, may indicate real-time stability margin information. To analytically explain the causes of the fluctuation, this paper first investigates the swing equation of a single machine-infinite bus (SMIB) system and then verifies the results by case studies on a large power system model. Section II explains why the OF fluctuates on a SMIB power system, which will be verified in section III. Section IV proposes a stability margin index based on the characteristics of OF fluctuation. Section V presents case studies on a two-generator system and a 179-bus system.

\section{ANALYSIS OF OSCILLATION FREQUENCY}

In this section, a second-order nonlinear differential equation for describing the rotor angle's behavior will be established from the swing equation of a SMIB. Then the characteristics of the OF will be discussed. Consider the swing equation of the SMIB power system following a disturbance:

$$
\left\{\begin{array}{l}
\Delta \dot{\delta}=\omega_{0} \Delta \omega_{r} \\
\Delta \dot{\omega}_{r}=\left(P_{m}-P_{e}-D \Delta \omega_{r}\right) / 2 H
\end{array}\right.
$$

where $\omega_{0}$ is the synchronous frequency, $\Delta \delta$ and $\Delta \omega_{\mathrm{r}}$ are the rotor angle and speed relative to their initial states $\delta_{0}$ and $\omega_{0}$, $P_{\mathrm{m}}$ and $P_{\mathrm{e}}$ represent the per-unit mechanic torque and electromagnetic torque, respectively. $H$ and $D$ represent the inertia and damping factor of the machine, respectively. Assume that $P_{\mathrm{m}}$ remains the same before and after the disturbance, i.e. ignore the effect of governor system.

$$
P_{m}=P_{\max } \sin \delta_{0} \quad P_{e}=P_{\max } \sin \left(\delta_{0}+\Delta \delta\right)
$$

where $P_{\max }$ is the maximum transfer power. Substitute (2) into (1) and eliminate $\Delta \omega_{\mathrm{r}}$, then get: 


$$
\Delta \ddot{\delta}+\alpha \Delta \dot{\delta}+\beta\left[\sin \left(\delta_{0}+\Delta \delta\right)-\sin \delta_{0}\right]=0
$$

where $\alpha=D / 2 H$ and $\beta=P_{\max } \omega_{0} / 2 H$ for simplicity. The solution $\Delta \delta(t)$ of (3) is determined given two initial conditions. Generally, if this SMIB power system remains stable following a disturbance, the solution $\Delta \delta(t)$ would oscillate for a period of time, such as several seconds or tens of seconds, and damp to zero eventually.

Equation (3) is a second-order nonlinear differential equation. When compared to the second-order ordinary differential equation, which has been well studied, the only difference is the third term. Thus, replace the third term using (4), then get (5).

$$
\begin{aligned}
& \sin \left(\delta_{0}+\Delta \delta\right)-\sin \delta_{0}=2 \sin (\Delta \delta / 2) \cos \left(\delta_{0}+\Delta \delta / 2\right) \\
& \Delta \ddot{\delta}+\alpha \Delta \dot{\delta}+\left(\beta \frac{\sin (\Delta \delta / 2)}{\Delta \delta / 2} \cos \left(\delta_{0}+\Delta \delta / 2\right)\right) \Delta \delta=0
\end{aligned}
$$

Recall that for the second-order ordinary differential equation (6), the natural oscillation frequency could be calculated by (7), where $a$ and $b$ are constants.

$$
\begin{aligned}
& \ddot{x}+a \dot{x}+b x=0 \\
& \omega_{n}=\sqrt{b}
\end{aligned}
$$

Comparing (5) with (6), we may conclude that the OF changes with the instantaneous value of $\Delta \delta(t)$ and the pre-fault power angle $\delta_{0}$. Under a certain power system operating condition, $\delta_{0}$ could be treated as a constant with respect to $\Delta \delta(t)$. If $|\Delta \delta(t)|$ is small enough to be negligible, the dynamic coefficient of $\Delta \delta$ in (5) will be close to a constant $\beta \cos \left(\delta_{0}\right)$ and hence $\omega_{\mathrm{n}}$ will degenerate into a constant, which is just the result from smallsignal stability analysis. Thus, we hypothesize that the instantaneous $\mathrm{OF}$ of $\Delta \delta(t)$ may be approximated as:

$$
\omega_{n}=\sqrt{\beta \frac{\sin (\Delta \delta / 2)}{\Delta \delta / 2} \cos \left(\delta_{0}+\Delta \delta / 2\right)}
$$

When $|\Delta \delta(t)|$ is not that small but bounded, by the continuity $\omega_{\mathrm{n}}$ will change continuously in an interval, say $\left[\omega_{\mathrm{nmin}}, \omega_{\mathrm{nmax}}\right]$.

Generally, the maximum deviation of $\Delta \delta(t)$ is proportional to the energy injected into the system by the disturbance. Then it will also determine the range of the OF by (8). Therefore, a disturbance with larger energy injected into the power system will enable the OF to change on a larger interval.

\section{VERIFICATION ON SMIB POWER SYSTEM}

To verify the changing OF phenomenon indicated by (8), a case study is performed on a SMIB system. Then a phaseplane trajectory (PPT) method from [9] is used to calculate the instantaneous OF and compare it to the Prony Method.

Let the parameters in (3) take these values: $D=1, \quad H=3$, $P_{\max }=1.7, \delta_{0}=20.8^{\circ}$, and $\omega_{0}=2 \pi \times 60 \mathrm{rad} / \mathrm{s}$. A line fault is applied at 1 second and the fault duration is increased to 0.33 second to push the system to its stability boundary, which generates a marginally stable case. Then, the system will take the following two initial values for the post-fault period and it is supposed to have large nonlinearity in the first several swings.

$$
\Delta \delta(0)=1.5171 \mathrm{rad} \quad \Delta \dot{\delta}(0)=8.2184 \mathrm{rad} / \mathrm{s}
$$

Fourth-order Runge-Kutta method is used to numerically solve (3) for $t=0 \sim 16 \mathrm{~s}$ as is shown in Fig. 1.

\section{A. Verification of Varying $O F$}

The verification of the fluctuation phenomenon in the $\mathrm{OF}$ will be done by contradiction. Assume that the OF is constant over time. Then data from the first 0.5 second of post-fault period are used to fit a damped sinusoidal curve using the model shown in (9) by the Least Mean Square approach. In (9), $A, \sigma, \omega$ and $\varphi$ are assumed to be constants. Similarly, another curve could be obtained using the last 5 seconds time window. The curve fitting results are shown in Fig. 1.

$$
\Delta \delta(t)=A e^{-\sigma} \sin (\omega t+\varphi)
$$

It can be seen from Fig. 1 that after 1 second, the optimal fitted curve using the first 0.5 second data of post-fault period has a slower oscillation than the numerical solution, while the curve from the last 5 seconds seems to have a faster oscillation in the beginning of the whole time widow. Thus, the constant OF assumption does not hold for the solution to the nonlinear swing equation. Furthermore, a simple conclusion could be drawn that in this stable case, the OF will start from a relatively small value and gradually increase as the angle difference is damped gradually.

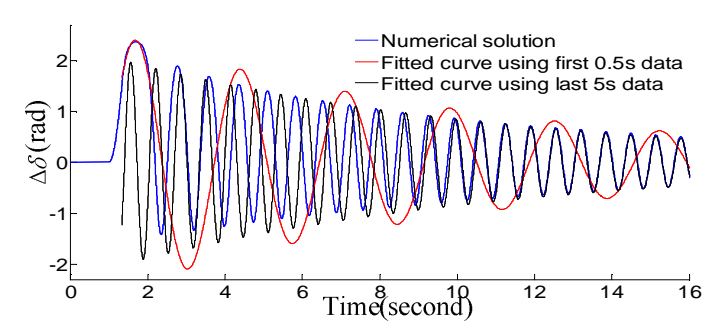

Figure.1.Numerical solution and its fitting curves

\section{B. Estimation of Instantaneous $O F$}

To validate the result implied by (8), the PPT method is used to estimate the OF with more sense of instantaneity to show how the OF fluctuates over time. An example of other techniques for real-time OF estimation is a Phase Locked Loop base $\mathrm{d}$ method [10]. The steps of the PPT method are:

i. Define $x(t)$ as (10) and calculate its derivative $x^{\prime}(t)$.

ii. Define $y(t)$ as (11).

iii. Draw the phase diagram using $(x(t), y(t))$ and calculate the point-wise OF by (12) and (13) using any two adjacent points.

$$
\begin{gathered}
x(t)=\Delta \delta(t) \\
y(t)=x^{\prime}(t) / \lambda, \lambda=\sqrt{\operatorname{var}(x(t)) / \operatorname{var}\left(x^{\prime}(t)\right)} \\
\alpha(t)=\left|\arctan \frac{|y(t+\Delta t)-y(t)|}{|x(t+\Delta t)-x(t)|}-\arctan \frac{|y(t)-y(t-\Delta t)|}{|x(t)-x(t-\Delta t)|}\right| \\
\omega(t)=\alpha(t) / \Delta t
\end{gathered}
$$


where $\alpha(\mathrm{t})$ is the angular displacement, $\omega(\mathrm{t})$ is the $\mathrm{OF}$ and $\Delta t$ is the sampling interval.

When applying the above approach to the solution shown in Fig. $1, \lambda$ and $\Delta t$ take 0.1 and $1.667 \mathrm{~ms}$, respectively in this case. The plot of $y(t)$ and the phase diagram of $x(t)$ are shown in Fig. 2 and Fig. 3, respectively. The OF is calculated using a two-point time window. Then perform the computation by moving the window point-wise to give the OF curve shown in Fig. 4. The OF estimated by (8) using data in Fig. 1 is also presented in Fig. 4. The Prony method is used to track the OF over a sliding time window slightly longer than the period of the swing. The tracked result is shown in Fig. 4 as well.

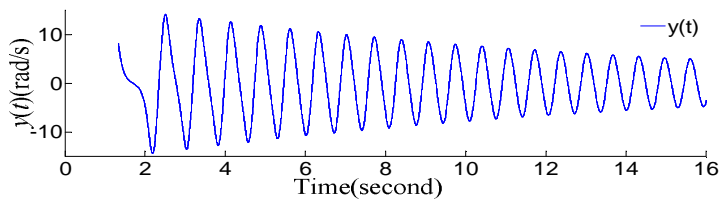

Figure.2.Plot of $y(t)$

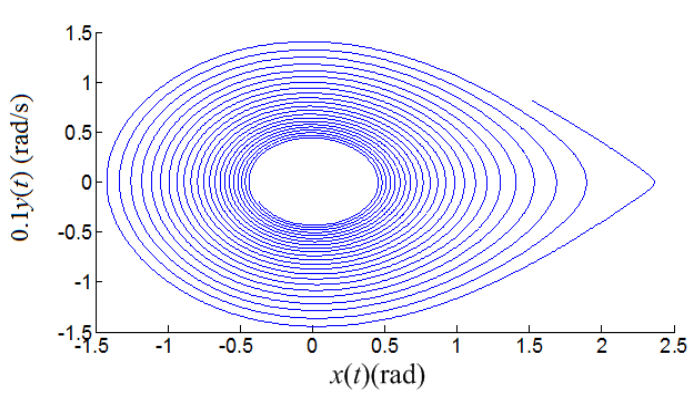

Figure.3.Phase diagram of $x(t)$

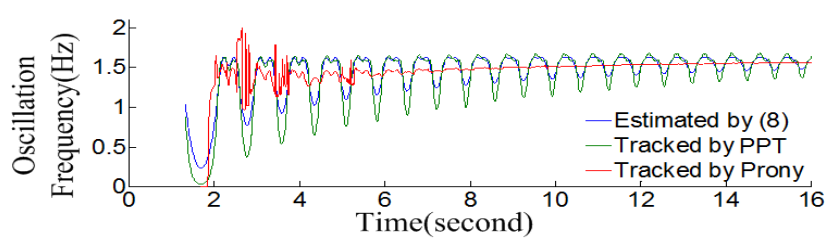

Figure.4.OF estimated by (8) and Tracked by the PPT and Prony

Fig. 4 shows the OF tracked by the three methods. Their general trends seem to match well, i.e. the range of the OF's fluctuation is big initially and gradually becomes smaller when the oscillation of angle difference is damped over time. The green curve by the PPT method exhibits more dynamics in the OF and matches the OF curve from (8) better. The red curve by the Prony method oscillates during the first 5 seconds and becomes stable after that. It does not show as much fluctuation because it uses data over a period of time to estimate average rather than instantaneous modal parameters.

\section{ANALYSIS OF STABILITY MARGIN}

This section is going to link the fluctuation of the OF to the system's stability margin (i.e. the distance to the angular stability boundary). Then, a stability margin index in terms of OF will be defined to indicate the stability level of a system when encountering a disturbance which excites significant oscillations. Finally, the impact from a change in the operating condition will be discussed.

\section{A. Stability Margin in Terms of $\mathrm{OF}$}

Based on (8), the OF may change with the instantaneous angle difference $\Delta \delta(t)$ and the initial operating condition, which depends on power angle $\delta_{0}$. Three interesting observations regarding the $\mathrm{OF}$ are discussed below.

Observation 1: The OF may change within a certain range over a post-disturbance period. Note that right after the disturbance, the system gets closest to the stability boundary and the OF has the largest fluctuations. When angle deviation $|\Delta \delta(t)|$ decreases, the system approaches a stable equilibrium and the OF fluctuation also decreases. Thus, the range of fluctuation may represent the criticality to instability.

Observation 2: Every maximum of $\Delta \delta(t)$, in Fig. 1, corresponds to a minimum of OF. This could be derived directly from the OF as a function of $\Delta \delta(t)$ by (8).

Observation 3: When the fluctuation range of the $\mathrm{OF}$ decreases, only the minima of the OF increase with time while the local maxima do not change significantly. Thus, the very beginning of OF following a disturbance, especially when its first minimum is reached, represents its maximum fluctuation.

A ccording to the above analysis, a Stability Margin Index (SMI) in terms of the OF is defined in (14).

$$
S M I=O F_{\min } / O F_{\max } \times 100 \%
$$

where $O F_{\min }$ and $O F_{\max }$ are the lowest and highest OFs captured following the disturbance. This index could be used to reflect the angular stability level:

- If $O F_{\min } \approx O F_{\max }$ over a monitored time window, then $S M I \approx 1$, which means that the system state during the time window is always near its stable equilibrium. Therefore, it is reasonable to say that the system has a relatively high stability level.

- If $O F_{\min } \ll<O F_{\max }$ over the time window, the system state has been far from the equilibrium sometime and hence its stability level should be relatively low, which is confirmed by a small $S M I$ value.

Remark: if the system loses angular stability during the time window, the definition of the OF after an unreturning instability point (a saddle point in the phase diagram) is unclear since that definition is extended from the small-signal stability point of view based on (6) and (7). However, we may hypothesize that $O F_{\min }$ must be far less than $O F_{\max }$, so SMI should be small even if it is not accurately zero.

By calculating the SMI using wide-area measurements acquired by phasor measurement units near key generators or on the boundary of the system's critical oscillation mode, the system operator could have a better awareness of how stable the system is following any disturbance. If a disturbance, although not causing instability, has SMI close to zero, it can be foreseen that the next disturbance of a bigger size may potentially cause instability. And, if the operating condition of the system becomes more stressed, then a disturbance of the same or even a smaller size may threaten stability. 


\section{B. Impact from a Change in the Operating Condition}

The SMI is able to foresee, especially from its value for the first few swings, how close the system has been to the boundary of stability during a disturbance. Note that the SMI is usually determined by the first minimum of the OF, which is corresponding to the first peak of $\Delta \delta(t)$. Thus, by changing $\delta_{0}$ in (8), which actually reflects the operating condition, the SMI may be changed. Fig. 5 shows the OF as a function of $\Delta \delta$ with different values of $\delta_{0}$. The intersection of OF and the $\Delta \delta$ axis represents how big $\Delta \delta$ is such that $\mathrm{OF}$ approaches zero, which indicates the system may lose its stability when reaching that point. By decreasing $\delta_{0}$, the intersection will move towards right, which allows $\Delta \delta$ to reach a larger value without losing stability. Thus, the system will be more stable under the same disturbance. Therefore, any change on the power flows that decreases the power output from the generator increases the stability margin.

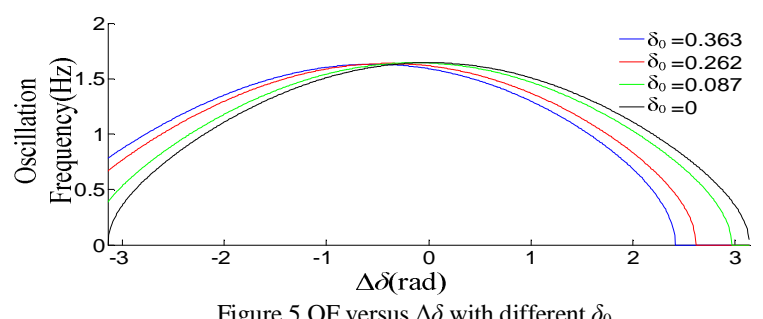

\section{CASE STUDIES}

This section shows case study results first on the twogenerator system shown in Fig. 6, which could be an equivalent of a power system with two areas of an interconnected power system, and then on a 179-bus simplified model of the WECC system. The purpose is to verify the prediction from (8) and show that the decrease in power transfer may enhance the stability of the system.

\section{A. Two-Generator Power System}

A temporary three-phase fault is applied to bus 1 at $t=1 \mathrm{~s}$. The fault clearing time is increased long enough, 0.597 second for this case, to generate a marginally stable case. After the fault is cleared, no line is tripped. Therefore, the system will come back to its original operation condition and OF will be computed by the PPT method to calculate the SMI. Since the active power is transferred from bus 2 to bus 1, thus increasing generation on bus 1 (PG1) and decreasing generation on bus 2 (PG2) can decreases the power angle $\delta_{0}$. Thus, PG2 is decreased from $80 \mathrm{MW}$ to $41 \mathrm{MW}$ by a step of $4 \mathrm{MW}$ while PG1 is increased to meet the balance of the power flow.

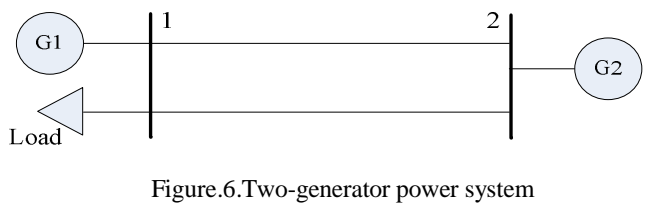

Fig. 7 shows the angle difference between G1 and G2. From Fig. 8 it can be seen that the OF increases from $0.12 \mathrm{~Hz}$, after the fault clearing time, to $0.65 \mathrm{~Hz}$, where the deviation of angle difference is small and the OF almost remains fixed. When PG2 takes different amounts of MW, the OF curves are shown in Fig. 9. By decreasing PG2 to 41MW, the SMI will increase from $20 \%$ to $54 \%$ if encountering the same disturbance in future, where Fig.10 shows time domain simulation result and compared to the original case.

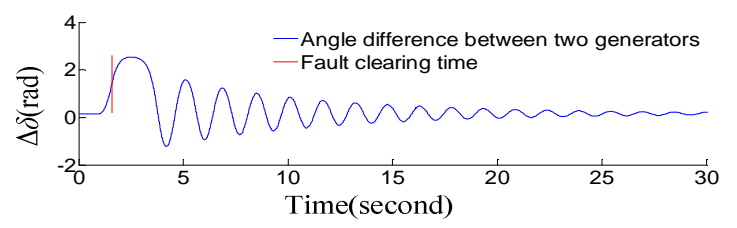

Figure.7.Angle difference between two generators
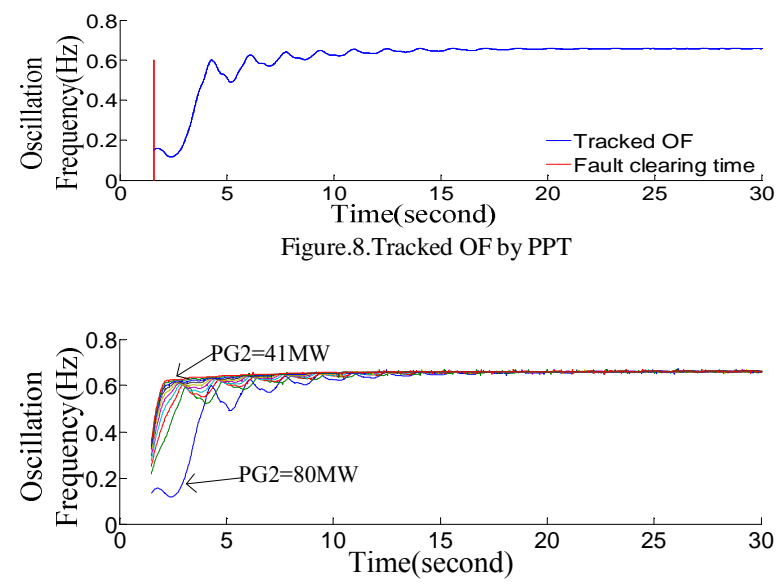

Figure.9.Tracked OF by PPT method with different PG2

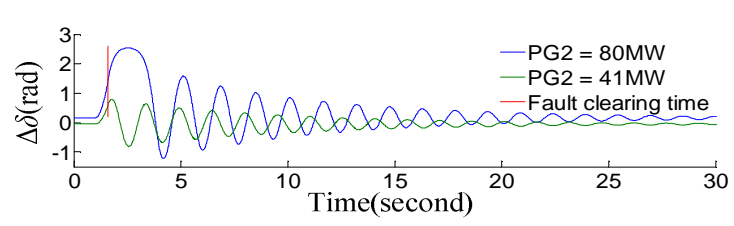

Figure.10.Influence of increasing PG2 on the oscillation

\section{B. 179-Bus Power System}

In the 179-bus power system shown in Fig. 11 with the following lines tripped before simulation: line 83-172, 83-170, 114-124 and 115-130, a three-phase fault on line 83-94 is added at 1 second, which is on a critical interface $0-1$, which corresponding to the famous California-Oregon Intertie (COI). This fault scenario is similar to the last fault in [9] with the only difference being that the fault clearing time is increased to its critical clearing time, i.e. 0.091 second, to make the system to be marginally stable. Fig. 12 shows the angle difference between two buses respectively in areas 0 and 1 . This angle difference is found to have two oscillation modes at about $0.15 \mathrm{~Hz}$ (mode 1) and $0.7 \mathrm{~Hz}$ (mode 2), respectively, as shown by its Fourier spectrum in Fig. 13. The $0.7 \mathrm{~Hz}$ mode appears only in the first 10 s and its amplitude is then damped to $<10 \%$ thereafter. In order to analyze how the frequency of each mode may change, low-pass filter and high-pass filter are applied to remove the other mode and noise, respectively, as shown in Fig. 12. The OFs estimated by the PPT method are shown in Fig. 14 and Fig. 15. The SMIs on two models are given in Fig. 16. 


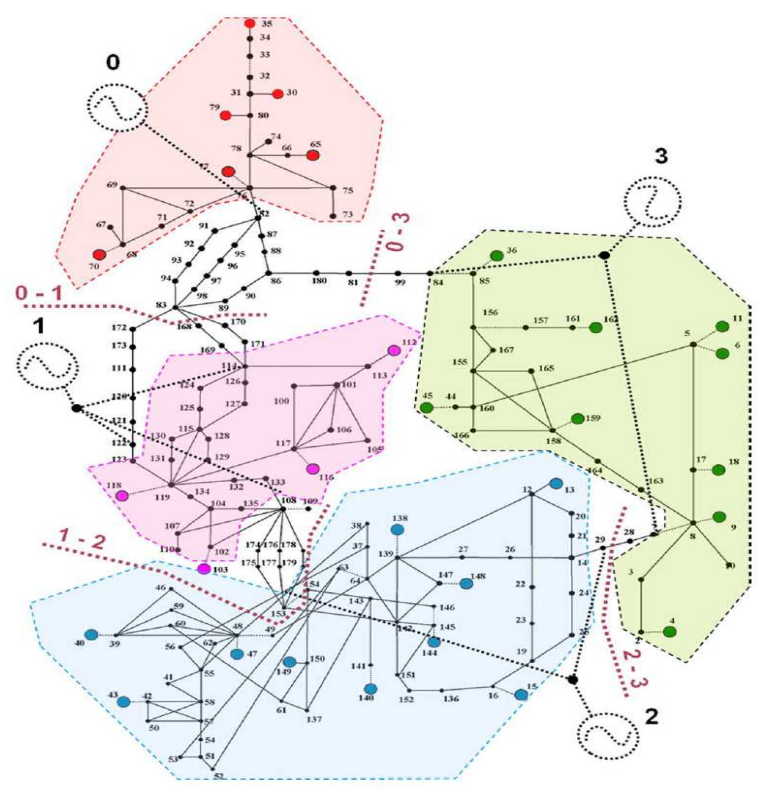

Figure.11.WECC 179-bus power system

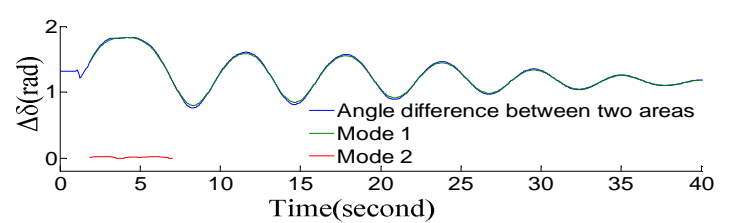

Figure.12.Angle difference between two areas

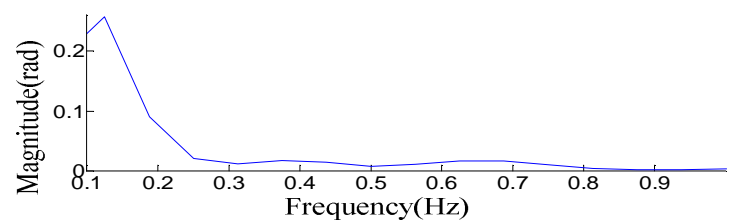

Figure.13.FFT analysis on angle difference

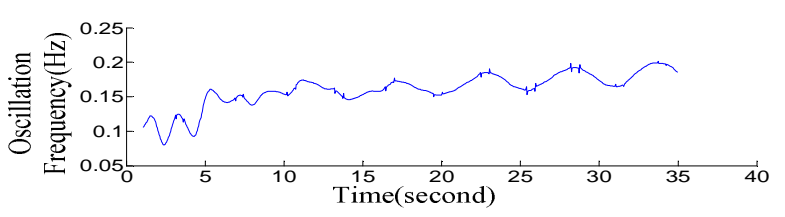

Figure.14.Tracked OF of Mode 1 by PPT

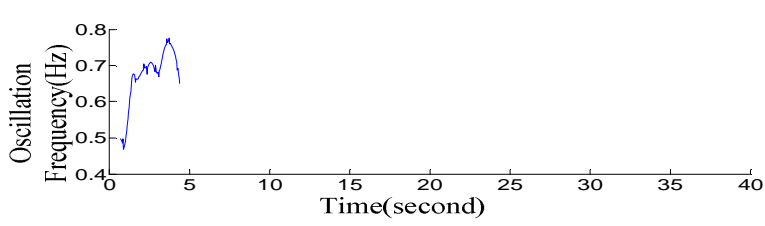

Figure.15.Tracked OF of Mode 2 by PPT

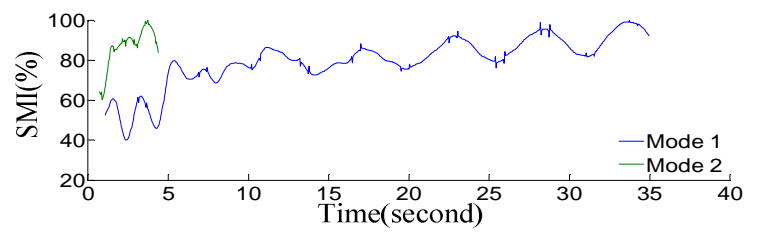

Figure.16.SMI on Mode 1 and Mode 2

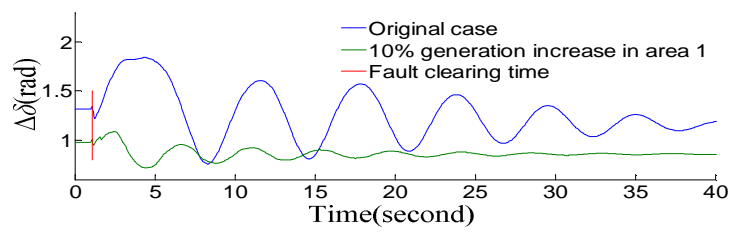

Figure.17.Influence of increasing generation in area1 on oscillation

Although there are two modes excited by the fault, mode 1 is more critical since it has more fluctuations than mode 2 . The lowest SMI for mode 1 is calculated to be $40 \%$. In an additional study, after a generation re-dispatch increases the active power generation in area 1 by $10 \%$ and decreases the same amount of generation in area 0 , the transferred power across the interface 0-1 will decrease and the lowest SMI is increased to $58 \%$. Time domain simulation results with and without that generation re-dispatch are shown in Fig. 17, from which the re-dispatched system is more stable. It confirms that the SMI can be an indicator of the stability level for taking early preventive actions.

\section{CONCLUSIONS}

This paper analyzes the fluctuation phenomenon of the oscillation frequency in power systems and proposes a stability margin index. Case studies have demonstrated that real-time monitoring the fluctuating OF can help estimate the angular stability margin and suggest preventive control.

\section{REFERENCES}

[1] K. Sun, K. Hur, P. Zhang, "A New Unified Scheme for Controlled Power System Separation Using Synchronized Phasor Measurements", IEEE Trans. Power Systems, vol. 26, No. 3, pp. 1544-1554, Aug. 2011

[2] K. Sun, X. Luo, J. Wong, "Early Warning of Wide-Area Angular Stability Problems Using Synchrophasors", IEEE PES General Meeting, 23-26 July 2012, San Diego, 2012

[3] A. Balanov, N. Janson, D. Postnov and O. Sosnovtseva, Synchronization: From Simple to Complex. Verlag Berlin Heidelberg: Springer, 2009, p. 21-73.

[4] The Power System Stability Subcommittee, "Identification of electromechanical modes in power systems," IEEE, San Diego, CA, TP462, June. 2012.

[5] P. Van Overschee, B. De Moor, Subspace Identification for Linear Systems: Theory, Implementation, Applications. Dordrecht, The Netherlands: Kluwer, 1996, pp.95-134.

[6] R. Brincker, et al. "Modal identification of output-only systems using frequency domain decomposition". Smart Materials and Structures, $10(3): 441$.

[7] J.L. Rueda, et al, "Wavelet-based analysis of power system lowfrequency electromechanical oscillations," IEEE Trans. Power Systems, vol. 26, No.3, pp. 1733-1743, August. 2011.

[8] S. A. Nezam Sarmadi, V. Venkatasubramanian. "Electromechanical mode estimation using recursive adaptive stochastic subspace identification," IEEE Trans. Power Systems, vol. 29, No.1, pp. 349358, January. 2014.

[9] K. Sun, et al, "An adaptive power system equivalent for real-time estimation of stability margin using phase-plane trajectories," IEEE Trans. Power Systems, vol. 26, No.2, pp. 915-923, May. 2011.

[10] K. Sun, Q. Zhou, Y. Liu, "A Phase Locked Loop-based Approach to Real-time Modal Analysis on Synchrophasor Measurements", IEEE Trans. Smart Grid, in press 